\title{
L'optimisation des prises d'eau et des stations de pompage
}

\author{
The optimization of intakes and pumping stations
}

par M. Milhe

ALSTOM Fluides et Mécanique

Centre d'Etudes et de Recherches de Grenoble

The high geometrical and functional diversity of the installations, dimensional optimization for cost reduction purposes, and necessity of validating the design and layouts, lead, in most cases, to the performance of tests on reduced scale models, for the water adduction and suction installations in particular.

The CERG establishment (Design and research center of ALSTOM Fluides et Mécanique) is definitely engaged in such types of experimental studies, to cope with the requirements of the Alstom company and its external customers.

The goal at stake is to optimize water collection from the following viewpoints :

- avoidance of vortex and air entrainment at pump inlet,

- homogeneous velocity profile in the suction manifold, and reduced fluctuations,

- absence of pre-rotation at pump inlet,

- study of the effects of constructive modifications.

\section{INTRODUCTION}

Les grandes centrales énergétiques, qu'il s'agisse de centrales thermiques classiques ou des îlots conventionnels des centrales nucléaires et de nombreuses implantations industrielles comportent des stations de pompage avec prise d'eau en mer ou en rivière.

L'importance du génie civil de ces ouvrages et les puissances mises en jeu justifient et nécessitent des études d'optimisation importantes pour rechercher la plus grande compacité des ensembles et sous-ensembles tout en conservant une bonne alimentation des composants et en particulier des pompes, pour réduire les pertes de charge des circuits et pour éviter les effets néfastes des régimes transitoires lors de la mise en route ou de l'arrêt volontaire ou accidentel des pompes.

La conception générale des ouvrages permet de distinguer trois grands ensembles dans les stations de pompage, ayant chacun leurs contraintes spécifiques : l'ouvrage d'amenée, l'ensemble filtre-pompe et le refoulement.

\section{II — OUVRAGES D'AMENÉE}

L'ouvrage d'amenée, qui peut être de deux types, à surface libre ou en charge, a pour mission d'assurer une distribution uniforme des débits sur les grilles, quels que soient les cas de fonctionnement envisagés (nombre de filtres, nombre de pompes en service, débits...) et les régimes hydrauliques (hautes eaux, basses eaux, vagues,...).
L'expérience permet généralement de dimensionner correctement les ouvrages d'amenée. La nécessité économique de compacter de plus en plus les ouvrages peut faire apparaitre des problèmes à la jonction ouvrage d'amenée - bassin d'aspiration (exemple fig.1), avec par exemple présence de vortex qu'il convient de supprimer si le bassin d'aspiration est de faible longueur et si les instabilités générées peuvent affecter le fonctionnement des équipements aval.

L'analyse porte également sur les régimes transitoires d'arrêt de pompes, en particulier l'arrêt accidentel et simultané de plusieurs pompes qui peut conduire à des débordements (exemple : figure 2) dans les ouvrages à surface libre ou à des surpressions-dépressions néfastes aux installations (ouvrages en charge).

\section{III —'ENSEMBLE FILTRES-POMPES}

Il a pour mission d'assurer une bonne alimentation des pompes, ce qui se traduit hydrauliquement, par :

- Une absence de vortex et d'entraînement d'air à l'entrée des tulipes de pompes,

- Un profil homogène de vitesse débitante dans la tulipe d'aspiration et des fluctuations réduites,

- Une pré-rotation limitée ou nulle à l'entrée des pompes,

- Un profil homogène des vitesses d'approche de l'eau dans les cellules de pompes,

- Une limitation des dépôts de sable, etc. 


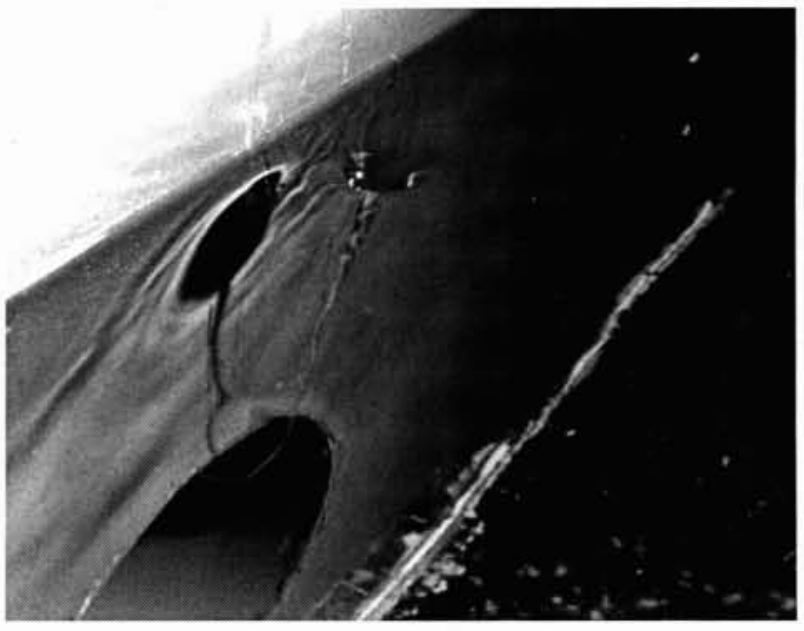

Ces caractéristiques doivent être vérifiées pour les multiples configurations d'exploitation résultant des combinaisons du nombre de filtres et de pompes en service et des variations de niveau dans les bassins.

Les essais sur maquette permettent de simuler facilement et rapidement ces différents cas, et de tester des améliorations (mise en place de guideaux, de croisillons, cônes ou écrans anti-vortex...) jusqu'à l'obtention de la qualité d'écoulement requise.

Deux schémas sont hydrauliquement possibles pour assurer la liaison entre filtre et pompe :

- l'un avec écoulement à surface libre,

- l'autre avec écoulement en charge.

La grande diversité géométrique ou fonctionnelle des aménagements, l'impossibilité de respecter totalement les recommandations habituelles ou les "standards ", l'optimisation des dimensions pour réduire les coûts de réalisation et la validation de la conception et des tracés conduisent à vérifier sur maquette à échelle réduite, le fonctionnement de l'ensemble de l'ouvrage.

Les phénomènes à analyser sur maquettes sont sous la dépendance des lois de similitude définies par les nombres de Froude, de Reynolds, de Weber, qu'il n'est pas possible de respecter simultanément, mais de nombreuses études ont montré que les modèles en similitude de Froude représentent correctement les phénomènes sous réserve d'une valeur suffisante des nombres de Reynolds et de Weber.

De ce fait, les études de prises d'eau sont réalisées sur des maquettes à des échelles qui peuvent être de l'ordre du 1/20 suivant les dimensions de l'installation réelle et de préférence au $1 / 15$ ou au $1 / 10$, ce qui permet par ailleurs de meilleures observations et des mesures plus précises et plus faciles des paramètres à contrôler. La plate-forme expérimentale (fig.3) comporte généralement trois faces transparentes (l'arrière et les latérales) facilitant les visualisations, et un fond transparent, au niveau des tulipes de pompes par exemple. Les parties sensibles de l'ouvrage qui nécessitent des observations particulières sont également réalisées en matériaux transparents.

Les différentes observations et mesures effectuées pour valider ou corriger le projet sont réalisées dans les différentes zones de l'équipement en particulier au niveau de la surface libre, pour caractériser les écoulements de surface

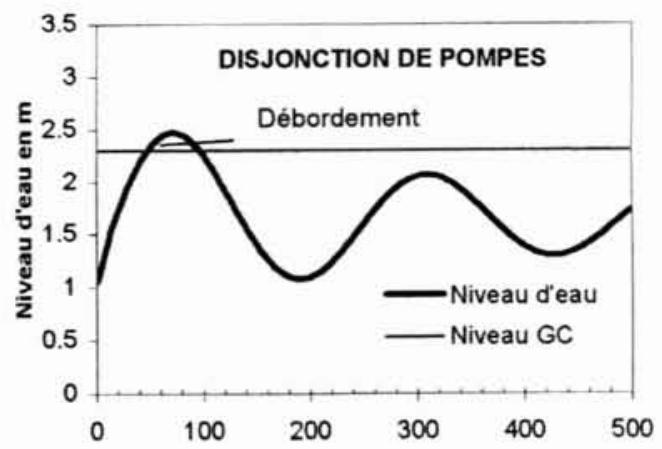

$\Delta$ 2. Fluctuations de niveau dans le bassin, à l'arrêt des pompes.

1. Entraînement d'air à l'amont du bassin d'aspiration.

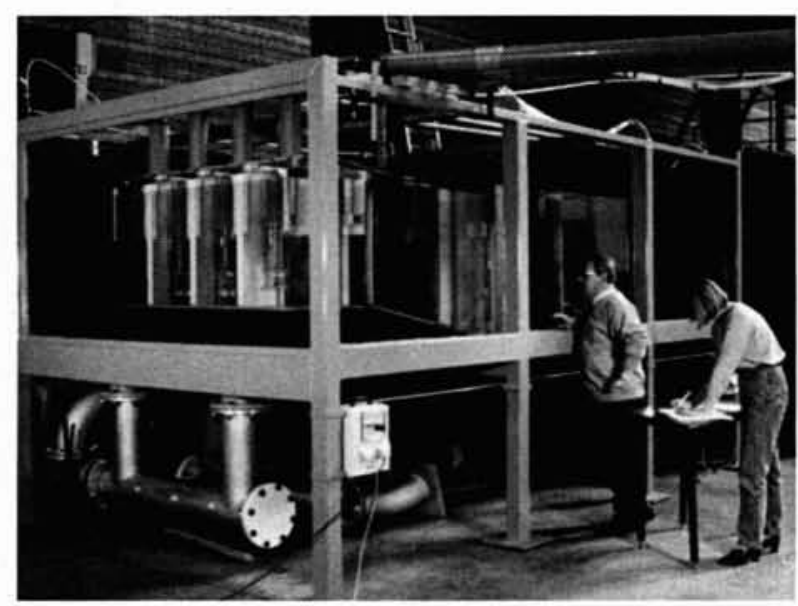

3. Plate-forme expérimentale.

(fig.4), analyser les effets de sillage d'éventuels obstacles amont et caractériser l'état et la stabilité de la surface libre.

Les analyses sont également réalisées au niveau de l'approche de l'écoulement vers les tulipes de pompes, en contrôlant les vitesses d'approche, ainsi que les dissymétries éventuelles, les risques de création de torches visualisables au moyen de colorant (fig.5) et de vortex établis avec entraînement d'air qui sont les phénomènes les plus graves et qui doivent, bien sûr, être proscrits.

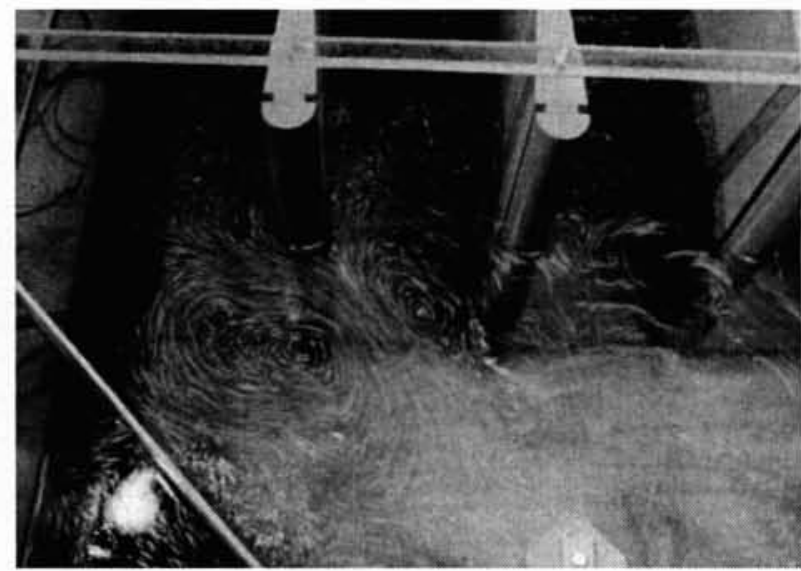

4. Tourbillons de surface dans le bassin d'aspiration. 


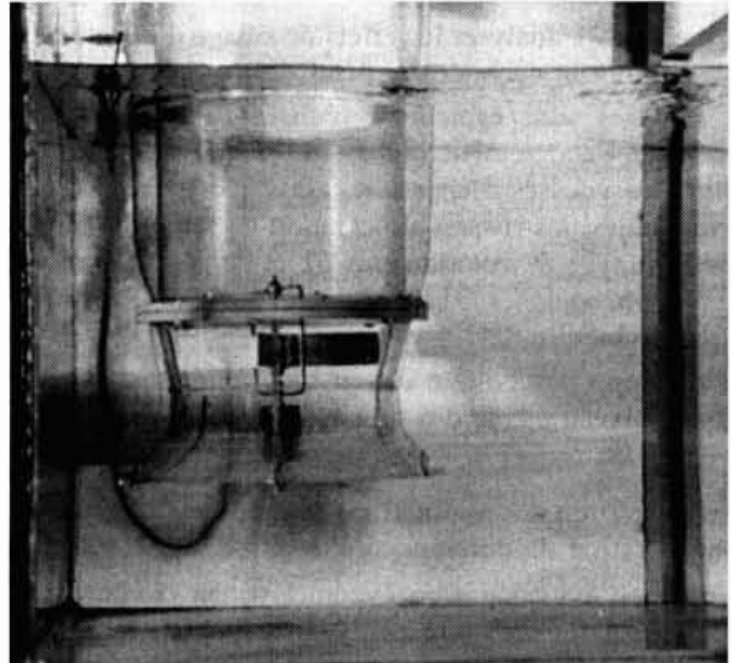

5. Rotation liquide sans entraînement d’air, visualisée par colorant.

Une attention particulière est portée aux écoulements au niveau même des tulipes d'aspiration de pompes avec :

- La visualisation des pré-rotations avec l'entrée de pompes, par injection de colorant (fig.6), utilisation de fils de laine... (fig.7),

- La recherche d'éventuels vortex cavitants en fond de bas$\sin$ (fig.6).

Les vortex et la cavitation qu'ils peuvent générer sont particulièrement préjudiciables au fonctionnement de la pompe et sources de bruit et de vibration (liés au collapse des bulles), de chute des performances (par obstruction partielle des canaux interaubes et modification de l'écoulement et de la hauteur engendrée), d'érosion et de dégradation du matériau.

Lorsque les manifestations visuelles d'un fonctionnement insatisfaisant de l'installation filtres -pompes ont été supprimées, il est nécessaire de vérifier que la pré-rotation d'angle $\alpha$ à l'entrée des pompes (avec tg $\alpha=$ composante tangentielle / composante axiale de la vitesse) reste inférieure à un seuil généralement compris entre 5 et $10^{\circ}$ : cette pré-rotation, mesurée au moyen de vorticimètres (fig.8) a de fortes répercussions sur le point de fonctionnement de la pompe concernée, et sa tenue mécanique (vibrations, érosion de cavitation (exemple figure 9)...).

La suppression de la pré-rotation passe par les tests de différentes solutions tels que croisillons, cônes, murets horizontaux et/ou verticaux... qui doivent rendre l'installation performante même dans le cas d'alimentation dissymétrique des équipements (exemples figures 10 et 11).

\section{LE REFOULEMENT DES POMPES}

Si le choix de la conception de la prise d'eau, de l'ouvrage d'amenée et de l'alimentation des pompes derrière les filtres est essentiel pour assurer un bon fonctionnement des pompes sans vibration, sans vortex et sans cavitation, la conception du refoulement des pompes est primordiale pour la sécurité des appareils situés à l'aval des pompes.

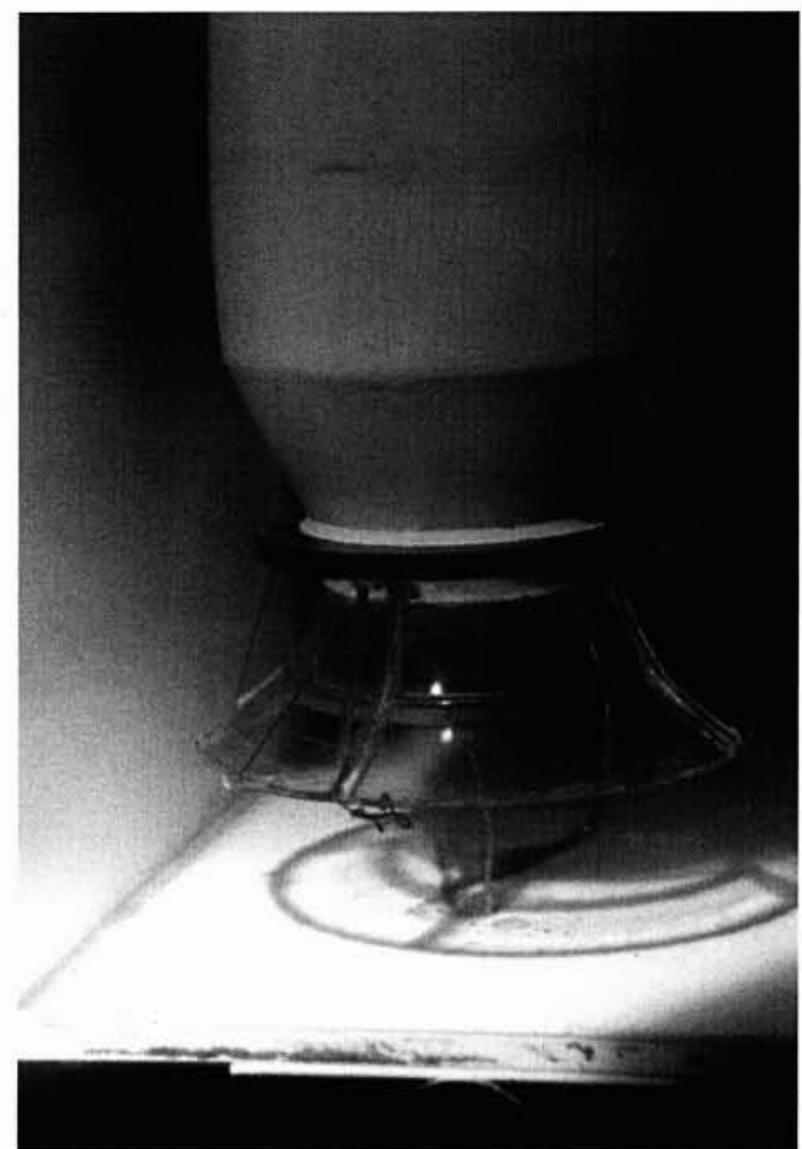

6. Vortex de fond de bassin et visualisation d'écoulement.
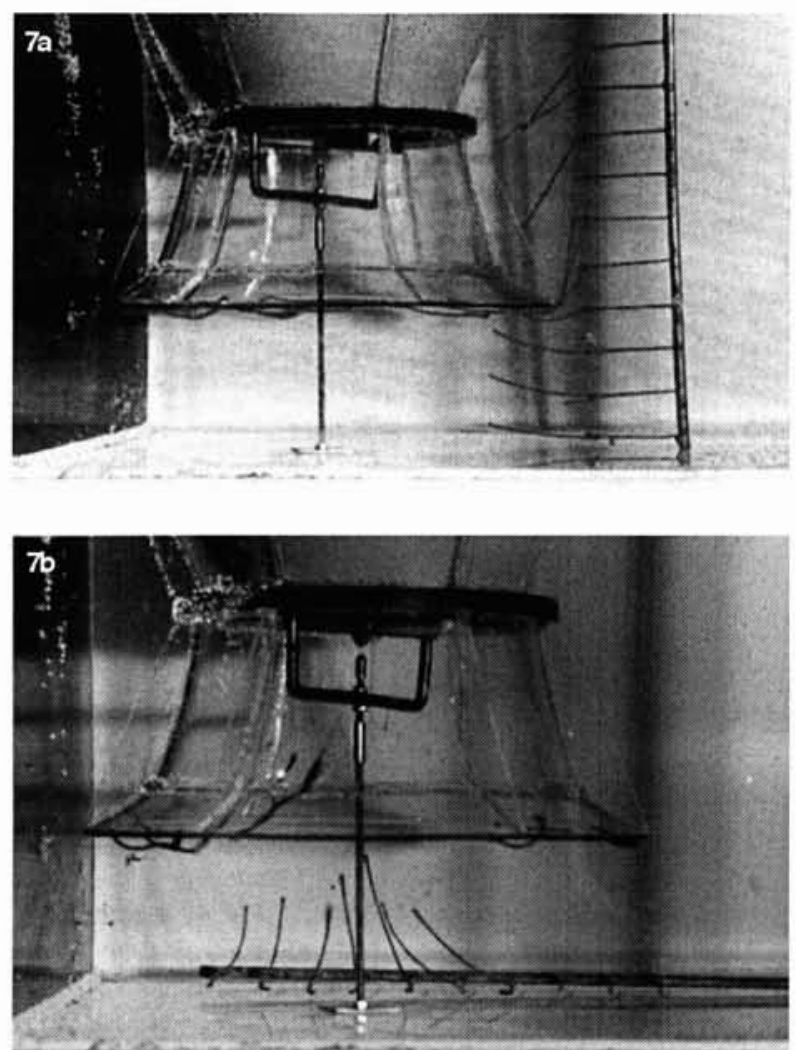

7 a,b. Ecoulements à l'approche de la tulipe, fils de laine. 


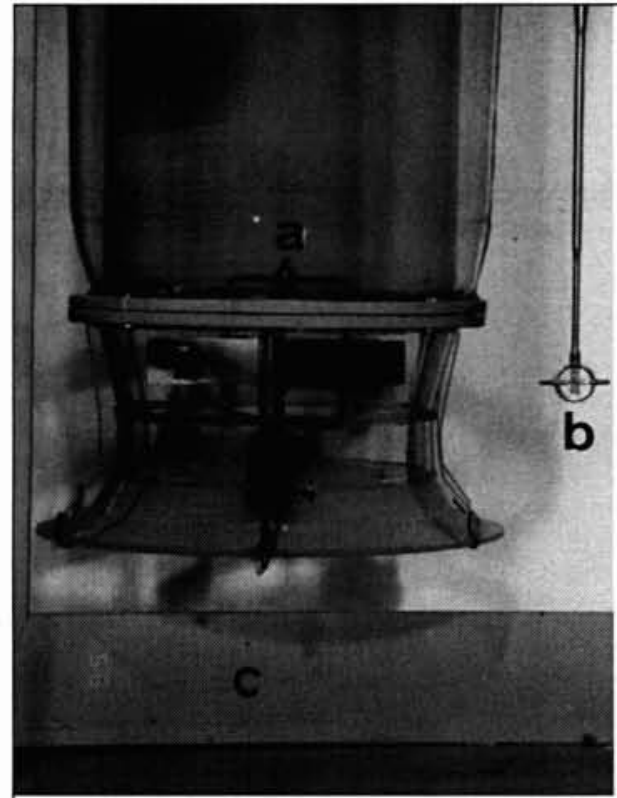

Fig. 8

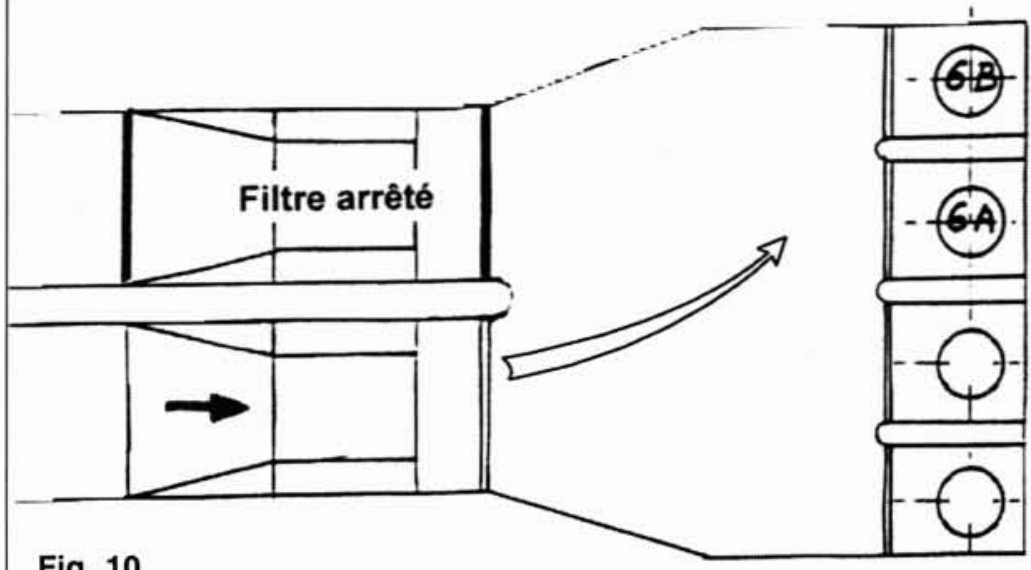

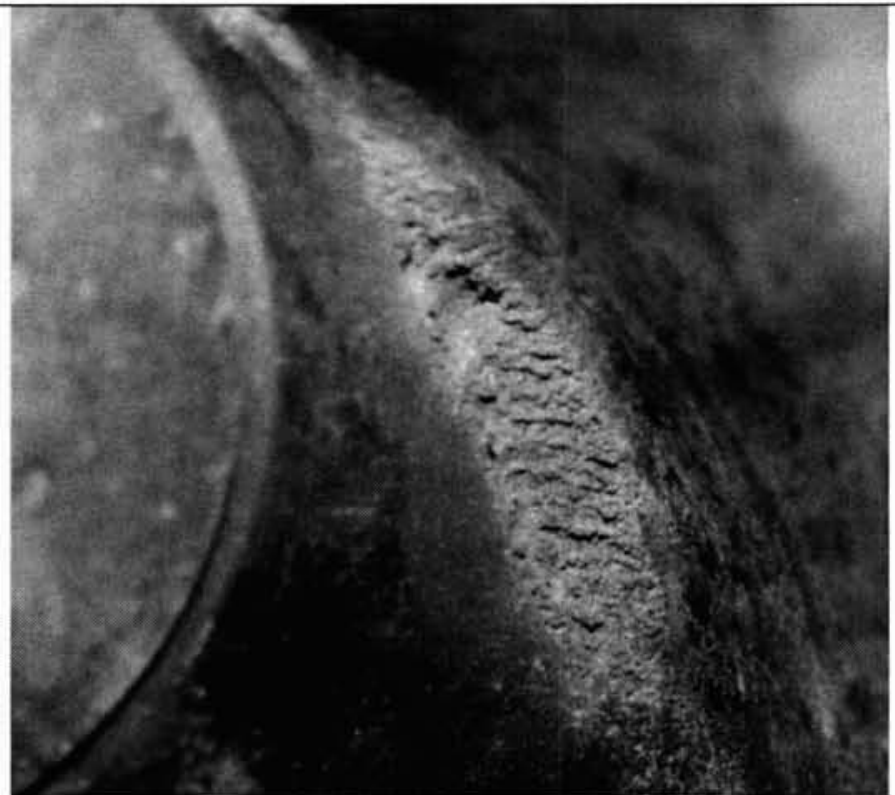

Fig. 9
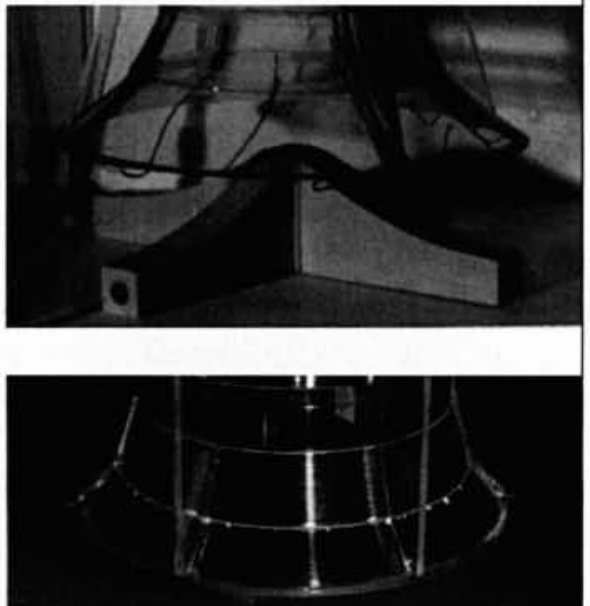

Fig. 11

8. Vorticimètre (a), mesure de la vitesse d'approche (b) et murets anti-vortex (c). 9. Constat de dégradation sur une pompe.

10. Exemple d'alimentation dissymétrique des pompes.

11. Installations de protection anti-rotation : croisillon (a), cône (b), cf. aussi fig. 8c.

Une étude de régime transitoire doit être associée aux conditions de fonctionnement de la station de pompage pour définir :

- Le type de robinetterie d'isolement anti-retour si nécessaire et ses lois d'ouverture et fermeture,

- Les accessoires de protection anti-bélier de la conduite, - Les surpressions résiduelles admissibles dans toutes les conditions d'exploitation normales ou accidentelles.

Ces études sont réalisées en utilisant des programmes de calcul basés sur la méthode des caractéristiques de L. Bergeron. Les valeurs de surpression et dépression dans les installations (exemple figure.12) sont liées à :

- La célérité du son dans les conduites,
- Le profil en long des installations,

- La longueur et le dessin des tuyauteries d'aspiration,

- L'inertie des groupes pompes,

- Le temps de fermeture et d'ouverture des vannes, etc.

La figure 13 montre les installations anti-bélier qui protègent les conduites de la station de pompage d'eau de mer du complexe chimique de Jorf Lasfar au Maroc. Trois réservoirs anti-bélier de $45 \mathrm{~m}^{3}$, chacun placé à l'aval immédiat des pompes et une cheminée d'équilibre de $38 \mathrm{~m}$ de hauteur en béton assurent ainsi la protection anti-bélier des installations de pompage et des trois conduites de refoulement de diamètre $2 \mathrm{~m}$ et de $1850 \mathrm{~m}$ de longueur. 


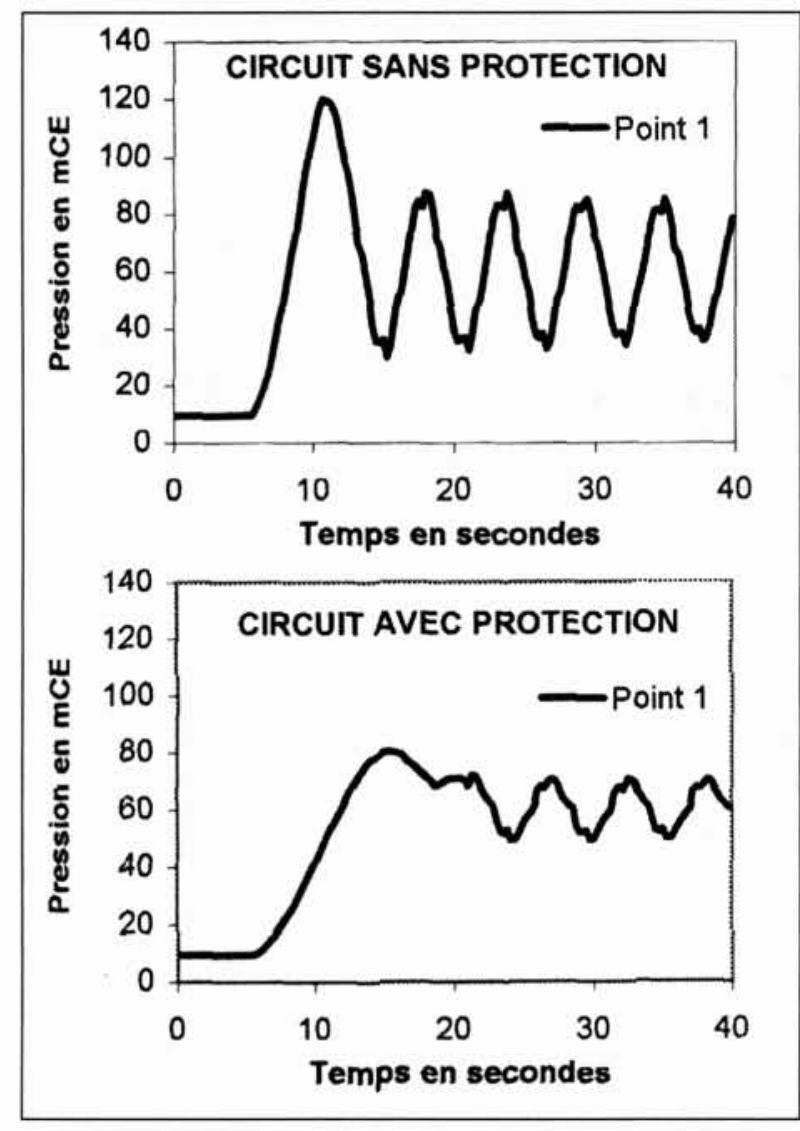

12. Pression aval résultant d'un arrêt de pompes sans (a) et avec (b) protections anti-bélier.

\section{$\mathrm{V} \square$ CONCLUSION}

C'est grâce à la complémentarité des simulations numériques et des simulations expérimentales sur maquettes que des installations hydrauliquement complexes, comme un ensemble prise d'eau - bassin de pompage - refoulement, peuvent être optimisées.

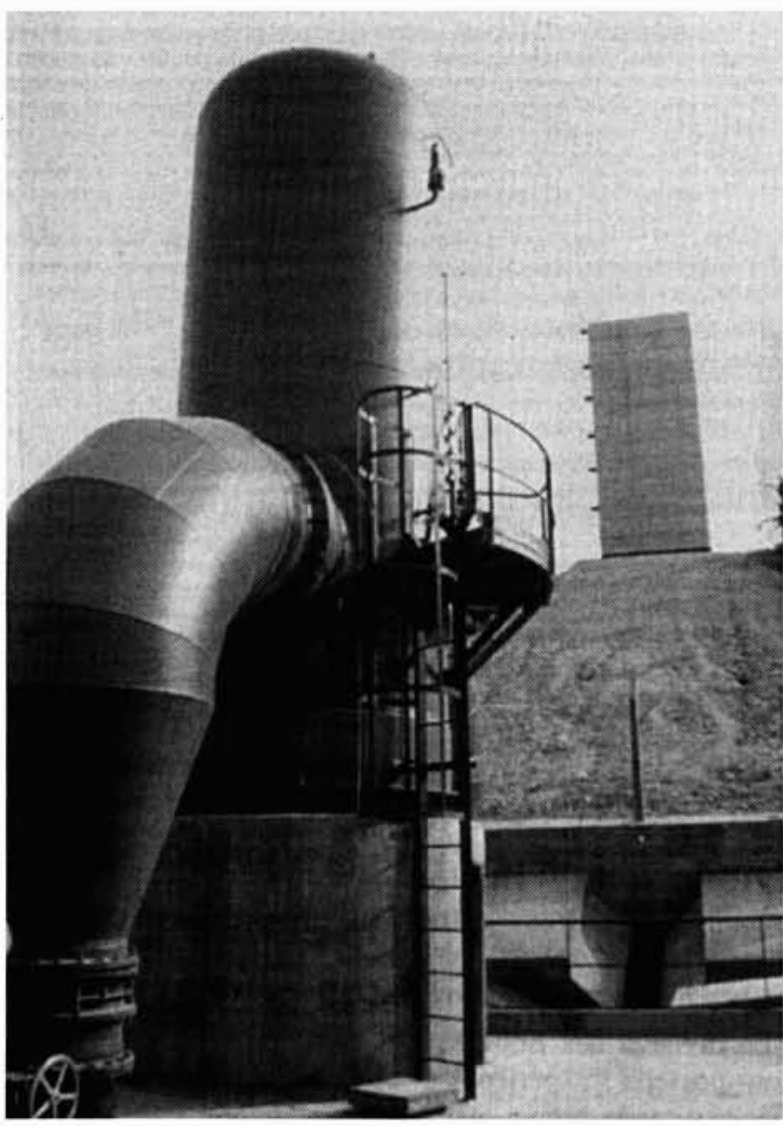

13. Installations de protection anti-coup de bélier.

Les choix techniques, l'originalité des solutions retenues, l'étude technico-économique incluant le coût du génie civil, le coût des équipements, d'exploitation et de maintenance permettent au consultant de comparer les différentes variantes et de garantir le fonctionnement de l'installation dans le respect du cahier des charges. 